\title{
Genome-Wide Association Studies Identified Resistance Loci to Orange Rust and Yellow Leaf Virus Diseases in Sugarcane (Saccharum spp.)
}

\author{
Xiping Yang, ${ }^{1}$ Sushma Sood, ${ }^{2}$ Ziliang Luo, ${ }^{1}$ James Todd, ${ }^{3}$ and Jianping Wang $1,4, \dagger$ \\ ${ }^{1}$ Agronomy Department, University of Florida, Gainesville 32610, U.S.A.; ${ }^{2}$ Sugarcane Field Station, U.S. Department of Agriculture- \\ Agricultural Research Service (USDA-ARS), Canal Point 33438, U.S.A.; ${ }^{3}$ Sugarcane Research Unit, USDA-ARS, Houma 70360, U.S.A.; and \\ ${ }^{4}$ Center for Genomics and Biotechnology, Key Laboratory of Genetics, Breeding and Multiple Utilization of Corps, Ministry of Education, \\ Fujian Provincial Key Laboratory of Haixia Applied Plant Systems Biology, Fujian Agriculture and Forestry University, Fuzhou 350002, \\ Fujian, China
}

Accepted for publication 27 September 2018

\begin{abstract}
Sugarcane (Saccharum spp.) supplies globally $\sim 80 \%$ of table sugar and $60 \%$ of bioethanol. Sugarcane orange rust and Sugarcane yellow leaf virus (SCYLV) are major sugarcane diseases, causing up to 50 and $40 \%$ yield losses, respectively. Sugarcane cultivars resistant to these diseases are needed to sustain sugarcane production in several regions. Dissecting DNA sequence variants controlling disease resistance provides a valuable tool for fulfilling a breeding strategy to develop resistant cultivars. In this study, we evaluated disease reactions to orange rust and SCYLV of a sugarcane diversity panel in repeated trials. We conducted a genome-wide association study between high-density markers and disease resistance
\end{abstract}

ABSTRAC

reactions. We identified 91 putative DNA markers and 82 candidate genes significantly associated with resistance to one of the two diseases. These provide an important genetic resource for finding genes and molecular markers for disease resistance. Our results emphasized the importance of utilizing a wide germplasm collection for breeding resistant sugarcane cultivars

Keywords: GWAS, population biology, Puccinia kuehnii, target enrichment sequencing
As an important economic crop, sugarcane (Saccharum spp.) is widely used for providing sugar and bioethanol, contributing $\sim 80 \%$ of sugar and $60 \%$ of bioethanol to the world (Dahlquist 2013). Grown in $>100$ countries, the crop is cultivated on $\sim 26.8$ million ha, with an annual harvest of 1.9 billion metric tons of sugarcane and a gross production value of $\$ 62.1$ billion (FAOSTAT 2016). To provide table sugar and bioethanol sustainably, sugarcane yield needs to be improved. However, there are various devastating diseases reported in sugarcane that substantially reduce sugarcane production and increase input costs owing to sophisticated disease management. Two diseases, sugarcane orange rust and Sugarcane yellow leaf virus (SCYLV), cause up to 50 and $40 \%$ yield losses, respectively (Débibakas et al. 2014; Magarey et al. 2011), in certain sugarcane-growing regions.

Sugarcane orange rust disease caused by a biotrophic fungus Puccinia kuehnii E. J. Butler is a major disease threatening sugarcane production in the United States (Yang et al. 2018a). Zhao et al. (2011) showed that orange rust disease could linearly reduce leaf Soil-Plant Analysis Development (SPAD) index, stomatal conductance, and transpiration rate, with the strongest impact on photosynthetic rate, showing physiological bases of sugarcane yield loss caused by the disease. Major reasons for destructive damage caused by the disease are the long duration of the disease because of the pathogen tolerating high temperature, that most sugarcane commercial cultivars are susceptible to the disease, and that efficient disease management methods are lacking. SCYLV is a

†Corresponding author: J. Wang; E-mail: wangjp@ufl.edu

Funding: This research is financially supported by the Florida Sugar Cane League and The Office of Science, U.S. Department of Energy Award DE-SC0006995.

*The $\boldsymbol{e}$-Xtra logo stands for "electronic extra" and indicates that five supplementary figures and three supplementary tables are published online.

The author(s) declare no conflict of interest.

(C) 2019 The American Phytopathological Society virus disease caused by an emerging virus evolving from Luteoviral and Poleroviral by recombination between them (Moonan et al. 2000). This virus is transmitted by aphids, mainly Melanaphis sacchari (Daugrois et al. 2011), and propagated by planting infected cuttings (Rassaby et al. 2004). The infection of the disease caused yellow leaf symptom, led to alterations in photosynthetic efficiency and carbohydrate accumulation (Gonçalves et al. 2005), and impacted sugarcane yield and juicy quality (Rassaby et al. 2003).

Like other crop diseases, the most economically and environmentally efficient way to control these diseases is to breed and plant diseaseresistant cultivars. Several studies have been performed in sugarcane to understand resistance to orange rust and SCYLV diseases. For example, three quantitative trait loci (QTLs) were identified for orange rust in a population of 173 progeny derived from a biparental hybridization (Yang et al. 2018a). A major quantitative trait allele and six markers were identified associated with SCYLV in a biparental population of 196 progeny (Costet et al. 2012) and a sugarcane cultivar panel $(n=189)$ (Débibakas et al.2014), respectively. In 2018, two QTLs controlling SCYLV resistance were identified, and together, they could reduce disease incidence by $31 \%$ (Islam et al. 2018). These studies improved understanding of the molecular resistance mechanisms to orange rust and SCYLV. However, these studies were conducted on either biparental populations or cultivar panels, and this limits the chance of identifying novel potential resistance loci owing to the narrow genetic basis of elite sugarcane cultivars. Limitations of these studies also included the low density of markers applied, no marker dosages, that interactions were considered in genome-wide association study (GWAS) models, and difficulty in transferring genotyping methods among different laboratories.

We evaluated disease reactions to sugarcane orange rust and SCYLV in a diversity panel with 308 accessions. The panel contained a substantial amount of the genetic variability present in the "World Collection of Sugarcane and Related Grasses" (WCSRG) and was applicable for comprehensive phenotypic and genotypic evaluation. Therefore, evaluating disease resistance to orange rust and SCYLV in this wide diverse collection of sugarcane 
and related germplasm could improve our chances of identifying useful and novel causal sequence variants. In addition, we characterized a large number of DNA sequence variants by deeply sequencing the coding regions of the panel through target enrichment sequencing (TES). We conducted GWAS by using GWASpoly (Rosyara et al. 2016) with high-density DNA markers and with marker dosages and interactions included in GWAS models. The results not only revealed the genetic bases of the disease resistance but also, provided important genomics resources for the sugarcane research and breeding community.

\section{MATERIALS AND METHODS}

Plants in the association panel. Based on genotypic evaluation (screened 36 microsatellite markers on 1,002 genotypes) and phenotypic evaluation (scored eight morphological traits three times in 1 year), a core diversity panel with 299 accessions was chosen to represent the genetic diversity of the WCSRG (Nayak et al. 2014; Todd et al. 2014). Nine additional sugarcane genotypes that were wildly grown commercial cultivars and/or frequently used as parents in sugarcane breeding programs were also included. Hence, the panel consisting of 308 accessions (Supplementary Table S1) was established at the U.S. Department of AgricultureAgricultural Research Service Sugarcane Field Station in Canal Point, Florida in 2013 in pots arranged in a randomized complete block design with three replicates for each accession. Luxury fertilizer and daily drip irrigation were used to keep plants healthy.

Disease reactions. For orange rust disease evaluation, artificial inoculation was conducted after a previously established protocol (Sood et al. 2009). In brief, we collected P. kuehnii urediniospores by vacuuming sugarcane symptomatic leaves, because the pathogen is a biotrophic fungus that is difficult to culture in medium. Freshly collected rust spores were prepared to a concentration of $10^{4}$ urediniospores per $1 \mathrm{ml}$ in a solution containing $0.1 \%$ (vol/vol) Tween-20 and $0.002 \% 1-$ nonanol. A 500- $\mu$ l suspension of the rust sporea was placed inside the leaf whorl of four stalks, and all three replicates of each accession were inoculated for each replicate. Four weeks after inoculation, symptoms of orange rust were scored from the inoculated plants using a scale from zero to four (Sood et al. 2009), with zero being fully resistant and four being fully susceptible. The sugarcane diversity panel was also tested for SCYLV infection by using a tissue blot immunoassay. Leaves from top visible dewlap leaves were collected randomly from all replicates of each accession. Immunoassay of blotted membrane was conducted as described (Schenck 1997).

A pairwise Pearson correlation among disease reactions of each disease reaction was estimated separately using the "Hmisc" package (Harrell 2018). Analysis of variance was conducted using the "nlme" package (Pinheiro et al. 2014). Broad sense heritability $\left(H^{2}\right)$ was calculated using the formula

$$
H^{2}=\sigma_{g}^{2} /\left(\sigma_{g}^{2}+\frac{\sigma_{e}^{2}}{r}\right)
$$

where $\sigma_{g}^{2}$ is the genetic variance among accessions, $\sigma_{e}^{2}$ is the residual variance, and $r$ is the number of replicates. For both diseases, the average disease reactions of three replicates were calculated for each accession and then, used for GWAS.

Sequence variant calling and GWAS. Young leaves were sampled from the panel for DNA isolation using the Cetyl trimethylammonium bromide (CTAB) method (Wang et al. 2010). The library construction and sequencing of TES were performed following previous reports (Song et al. 2016). In brief, 60,000 120-bp probes were designed according to the sorghum genome (Paterson et al. 2009). The raw reads, generated by Illumina HiSeq 2000, were trimmed using Trimommatic v0.36 (Bolger et al. 2014) to remove sequencing from ends of reads with a Phred quality score $<20$. Clean reads were mapped to the sorghum genome v3.0 (Paterson et al. 2009) using BWA-mem (Li 2013) with default settings. Singlenucleotide polymorphisms (SNPs) and insertion and deletions (InDels) were called using Unified Genotyper implemented in Genome Analysis Tool Kit v3.30 (McKenna et al. 2010). The raw sequence variants were subjected to multiple filtering for each sample to ensure that accurate genotypes were called (Yang et al. 2017, 2019). Then, genotypes of sequence variants (SNPs and InDels) were scaled to the ploidy 12 based on the ratio of reads.

The population structure of the panel and the GWAS were performed following previously established methods (Yang et al. $2018 b$ ). In brief, we used discriminant analysis of principal components (DAPC) to assess the population structure of the panel (Jombart 2008; Jombart et al. 2010). The GWAS was run using GWASpoly (Rosyara et al. 2016) to take gene actions into consideration. We performed GWAS runs on the full panel in addition to four subpopulations to maximize the association identification (details are in Yang et al. 2018b). Sequence variants (SNPs and InDels) used in the GWAS were further filtered based on sample call rates $(\geq 95 \%)$ and minor allele frequency (MAF $\geq 0.05)$. Eight samples with $>20 \%$ missing data were removed from subsequent analysis. The annotations of candidate genes were downloaded from the sorghum genome reference (Paterson et al. 2009), and their deduced domains were further annotated by InterProScan 5.0 (Jones et al. 2014).

\section{RESULTS}

Sequence variants and subpopulations of the panel. The sequence variants were called following previous methods (Yang et al. 2018b). After stringent filtering, 74,900 common markers (MAF $\geq 0.05$ and call rate $\geq 95 \%$ in the diversity panel of 308 samples) were obtained, including 65,000 SNPs and 9,900 InDels. The 74,900 markers were used for the GWAS with an average marker distance of $9.1 \mathrm{kbp}$ according to the sorghum genome. Six subpopulations were identified in this panel, including four Saccharum species and two non-Saccharum species groups, which were Saccharum spontaneum (101 accessions), Saccharum officinarum (47 accessions), modern Saccharum hybrids (114 accessions), ancient Saccharum hybrids (17 accessions), the genus Miscanthus (10 accessions), and the genus Erianthus (19 accessions). This group assignment was supported by biological classification of this panel from the WCSRG, and then, it was used for the GWAS to control the effect of population structure.

We investigated sequence variants in six subpopulations of the diversity panel. Five accessions were selected from each subpopulation based on their genetic distance and sequence depth. Among the 30 accessions, 1.04 million SNPs were characterized (MAF $\geq 0.05$ and call rate $\geq 90 \%$ ). For each subpopulation, the number of SNPs ranged from 209,140 (the genus Erianthus) to 404,061 (modern Saccharum hybrids) (Table 1). The numbers of SNPs in Saccharum hybrids (modern Saccharum hybrids and ancient Saccharum hybrids) were higher than their ancestries, $S$. spontaneum and S. officinarum, indicating that the interspecies hybridization resulted in an increase of genetic diversity. We also surveyed sequence variants in 35 nucleotide binding site-leucinerich repeat (NBS-LRR) genes (Yang and Wang 2016), which were covered by at least $600 \mathrm{bp}$ in coding regions by our TES data. Overall, 3,003 SNPs were identified in the 35 NBS-LRR genes, with the number of SNPs for each gene ranging from 3 to 362 . The SNPs in NBS-LRR genes in the six subpopulations showed similar patterns as the SNPs at a genome-wide scale (Table 1). We calculated the number of SNPs in NBS-LRR genes per 1,000 SNPs at a genome-wide scale. The mean number was 2.88 , ranging from 2.81 (the genus Miscanthus) to 3.98 (the genus Erianthus) (Table 1). In the genus Saccharum, S. spontaneum had the highest number (3.68), whereas the Saccharum hybrids were between the two 
ancestors, and modern Saccharum hybrids were closer to S. officinarum (3.06).

The percentage of heterozygous SNPs among the total SNPs was significantly different among the six subpopulations at a genomewide scale $(P<0.0001)$ and in NBS-LRR genes $(P<0.05)$ (Fig. 1A). At a genome-wide scale, the genus Miscanthus had the highest percentage of heterozygous SNPs (33.28\% on average), whereas the genus Erianthus had the lowest (13.44\% on average). Both modern Saccharum hybrids and ancient Saccharum hybrids showed increased percentages of heterozygous SNPs compared with S. spontaneum and S. officinarum. Similar patterns were observed for percentages of heterozygous SNPs in NBS-LRR genes. However, the percentages of heterozygous SNPs in NBS-LRR genes were significantly higher than those at a genome-wide scale $(P<0.001$ for all pairwise comparisons). The average percentages of heterozygous SNPs ranged from $57.07 \%$ (the genus Erianthus) to $76.13 \%$ (the genus Miscanthus), two to four times higher than those at a genome-wide scale. The ratios of missense effect to silent effect SNPs at a genome-wide scale were close to one (Fig. 1B). However, this ratio significantly deviated from one in NBS-LRR genes ( $P<0.001$ for all pairwise comparisons), indicating that there are more SNPs with missense effects in these putative resistance genes. The average ratio was 1.42, ranging from 1.24 (the genus Erianthus) to 1.60 (modern Saccharum hybrids and ancient Saccharum hybrids). The percentages of heterozygous SNPs and the ratio of SNPs with missense effect to those with silent effect were significantly different in subpopulations, showing unique species sequence features because of evolution or selection pressure. In addition, the percentages of heterozygous SNPs and the ratio of SNPs with missense effect to those with silent effect were dramatically increased in NBS-LRR genes, indicating that new alleles were quickly generated in these disease resistance genes.

Disease reactions to orange rust and SCYLV diseases. We evaluated disease reactions to orange rust and SCYLV for all entries in the panel. The reactions of the three replicates were significantly correlated with mean correlation coefficients of 0.52 and 0.58 for orange rust and SCYLV, respectively $(P<0.0001)$, indicating good repeatability of phenotypic disease evaluation. We also investigated yield components on the same diversity panel (Yang et al. 2018b) and found that the disease reactions to orange rust and SCYLV were significantly related to stalk number, stalk diameter, and leaf width $(r>0.5, P<0.0001)$, suggesting that both diseases substantially impact yield components. The broad sense heritability values were 0.76 and 0.80 for the resistance to orange rust and SCYLV, respectively, suggesting that resistance to each disease was largely controlled by genetic factors. The mean disease reactions in the diversity panel to orange rust and SCYLV segregated well (Fig. 2A and B), and they were used for subsequent GWAS.

The disease reactions were significantly different among the six subpopulations for both diseases $(P<0.0001)$ (Fig. $2 \mathrm{C}$ and D). $S$. officinarum was highly susceptible, with average disease reactions of 2.1 and $87.2 \%$ for orange rust and SCYLV, respectively. $S$. spontaneum and two non-Saccharum species showed good resistance to both diseases compared with S. officinarum. No difference of disease reactions to both diseases was observed for modern Saccharum hybrids and ancient Saccharum hybrids. However, in responding to orange rust, resistance of modern Saccharum hybrids was more similar to that $S$. officinarum, whereas ancient Saccharum hybrids were closer to $S$. spontaneum (Fig. 2C).

GWAS on orange rust and SCYLV resistance. After applying a rigorous threshold $(P<0.05$ after Bonferroni correction), we identified 65 and 26 nonredundant markers significantly associated with the two diseases orange rust and SCYLV, respectively (Figs. 3 and 4, Supplementary Figs. S1 and S2, Supplementary Tables S2 and S3). The naivve $P$ values to claim significance ranged from $9.12 \mathrm{E}^{-7}$ to $5.75 \mathrm{E}^{-6}$ depending on GWAS models. The association rate of sequence variants was $0.55 \%$ (91/ $74,935=0.12 \%$ ). The Q-Q plots of GWAS supported that DAPC could reduce statistical inflation owing to population structure for all models, except for some suspicious inflations observed under the general model in the GWAS in $S$. spontaneum (Supplementary Figs. S3 and S4). To validate whether the suspicious inflations because of population structure existed in $S$. spontaneum, we carefully reassessed population structures in this group using DAPC and phylogenetic analysis. The results confirmed that miniature structures existed in this group (Supplementary Fig. S5), which could likely be neglected if the same analysis was done in the whole diversity panel. However, the GWAS, including the new population structures, had almost the same results as before $(93.3 \%$ of the markers were common between the two GWAS results). Therefore, we conclude that the population structures in $S$. spontaneum probably did not affect the GWAS in this study. The numbers of associated markers were $11,8,76,1$, and 5 for the full population and subpopulations $1,2,3$, and 4 , respectively. The majority of the markers $(83.5 \%)$ identified by the GWAS were in S. spontaneum, a wild progenitor species of sugarcane considered to be a major contributor to stress tolerance of modern sugarcane hybrids. Seven markers were identified in at least two runs of the GWAS (1ind22220477, 1ind57588903, and 10P4035066 for orange rust and 2P5229444, 2P7391840, 4P64528633, and 8P55553144 for SCYLV).

Among the 91 associated DNA markers, 84 were in genic regions of 70 gene models, and 7 were in intergenic regions based on the sorghum genome. Among the markers in gene models, 34 landed in coding regions, and 24 caused nonsynonymous mutations, whereas the remaining 50 markers were distributed in three-primer untranslated regions (UTRs) (15) and intron regions (35). To further investigate the genes associated, a total of 82 genes were obtained by including 70 genes containing associated markers and 12 genes flanked by associated markers. Therefore, the 82 nonredundant genes were considered as candidate genes associated with at least one of two diseases evaluated in the diversity panel. By searching enriched Gene Ontology and pathway analysis (KEGG) (Yi et al. 2013), the category of sulfur relay system was enriched in the genes significantly $(P<0.05$ adjusted for false discovery rate) associated with SCYLV. Based on annotations and predicted domains, the associated genes were diverse in functions, including transcription factors, protein kinases, transporters, and receptor.

We focused on 16 genes containing nonsynonymous mutations by searching their gene ontology annotations (Lyne et al. 2015). We found nine genes regulating gene expression (four transcription

TABLE 1. Summary of single-nucleotide polymorphisms (SNPs) at a genome-wide scale and in nucleotide binding site-leucine-rich repeat (NBS-LRR) genes in the diversity panel

\begin{tabular}{|c|c|c|c|}
\hline Subpopulation & Number of SNPs (genome wide) & Number of SNPs (NBS-LRR) & $\begin{array}{l}\text { Ratio of SNPs (NBS-LRR/ } \\
\text { genome wide } \times 1,000)\end{array}$ \\
\hline Saccharum spontaneum & 340,683 & 1,255 & 3.68 \\
\hline Saccharum officinarum & 301,347 & 923 & 3.06 \\
\hline Modern Saccharum hybrids & 404,061 & 1,288 & 3.19 \\
\hline Ancient Saccharum hybrids & 394,893 & 1,329 & 3.37 \\
\hline Genus Erianthus & 209,140 & 833 & 3.98 \\
\hline Genus Miscanthus & 360,705 & 1,012 & 2.81 \\
\hline Total & $1,042,338$ & 3,003 & 2.88 \\
\hline
\end{tabular}


factors [Sobic.001G365332, Sobic.003G109900, Sobic.006G173500, and Sobic.007G165100], three involved in chromosome remodeling [Sobic.007G096900, Sobic.008G129700, and Sobic.009G245400], one eukaryotic initiation factor [Sobic.003G129500], and one involved in transfer ribonucleic acid (tRNA) processing [Sobic.003G434700]). Seven genes were important components for biological processes through enzymatic activity (four genes, including a leucine-rich repeat and protein kinase [Sobic.005G057700], a sugar transferase [Sobic.002G357900], a cellulose [Sobic.010G130300], and a phosphogluconate dehydrogenase [Sobic.010G106900]) and receptor activity (three genes, including a bacterial extracellular solute binding protein [Sobic.010G068800], a protein binding [Sobic.006G011300],
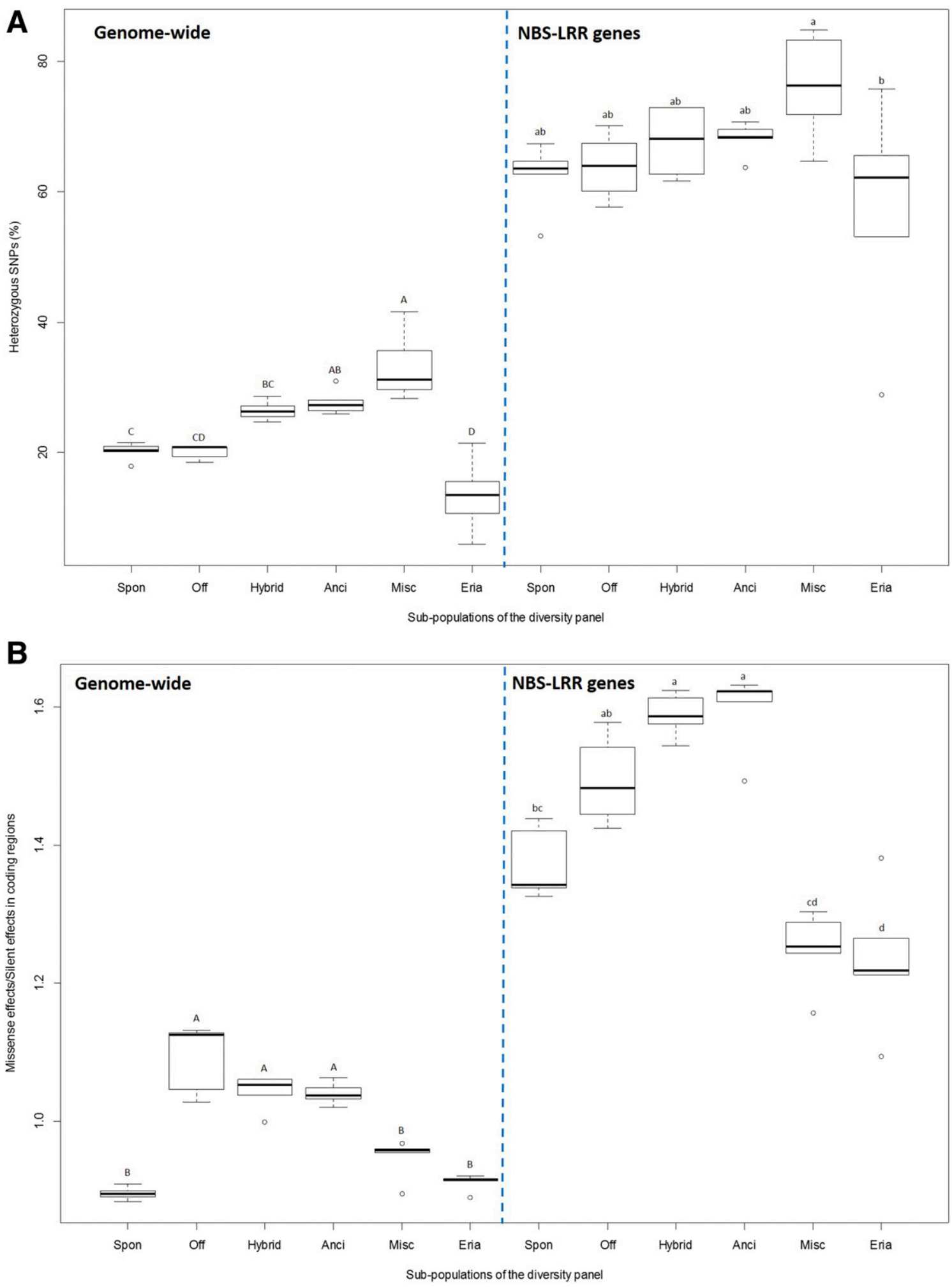

Fig. 1. Single-nucleotide polymorphisms (SNPs) in the subpopulations of the diversity panel. A, Percentage of heterozygous SNPs at a genome-wide scale and in nucleotide binding site-leucine-rich repeat (NBS-LRR) genes. B, Missense effect/silent effect SNPs in coding regions. Anci, ancient Saccharum hybrids, including Saccharum barberi and Saccharum sinence; Eria, the genus Erianthus; Hybrid, modern Saccharum hybrids; Misc, the genus Miscanthus; Off, Saccharum officinarum; Spon, Saccharum spontaneum. Tukey HSD test was applied to compare mean value of parameters of all groups at a genome-wide scale and NBS-LRR genes, respectively. Different letters indicate significant different level at $P<0.05$. 
and a lipid binding [Sobic.006G011300]), reflecting the molecular mechanisms of these genes in responding disease infection. Interestingly, the marker (8P55553144) in the gene (Sobic.008G129700) was identified to be associated with SCYLV by two different models. This nonsynonymous mutation happened at the first nucleotide of its codon, and it caused an amino acid to change from valine to phenylalanine. The disease reactions to SCYLV were significantly different between the two genotypes of the SNP (the average infection rate was $69.2 \%$ for homozygous reference allele [CC] and $22.6 \%$ for heterozygous genotypes [CT]; $P<0.0001$ ) (Fig. 5). The gene containing an alternative allele $(\mathrm{T})$ was resistant to the disease and showed a dominance effect to the gene having the reference allele (C).

\section{DISCUSSION}

A useful strategy for breeders to develop resistant cultivars is to dissect underlying loci controlling disease resistance. In this study, we used a previously developed representative sugarcane panel, applied high-density DNA markers, and included dosages and allele interactions into GWAS analyses; 91 putative DNA markers and 82 genes significantly associated with orange rust and SCYLV resistance were identified. This research not only identified valuable and novel genomic resources but also, emphasized the importance to utilizing a wide range of germplasm for identifying genetic resources of disease control.
Disease resistance to orange rust and SCYLV in sugarcane. Three QTLs controlling orange rust resistance in sugarcane (Yang et al. 2018a) were also detected in this association study. The major QTL (qORR109) was located at $\sim$ Chr02:61.2 $\mathrm{Mbp}$, with a logarithm of odd $=19.3$ and phenotypic variance explained $=58 \%$. In our study, two InDels (2ind55651651 and 2ind57730875) and three SNPs (2P68087100, 2P68782010, and 2P71872434) were discovered to be strongly associated with the disease $(-\log [P]$ ranging from 5.49 to 7.26 , with a distance $\sim 10$ Mbp [3.4 to $10.7 \mathrm{Mbp}$ ] from its closest flanking marker of qORR109). More interestingly, the InDel 2ind55651651 was in a gene (Sobic.002G176700) belonging to leucine-rich repeat receptor-like kinases, which were involved in defending the host against pathogens (Diévart et al. 2011). In addition, the distances of the closest associated markers in this study to the two minor QTLs, qORR4 and qORR102, were 8.3 and $2.7 \mathrm{Mbp}$, respectively, with $-\log (P)$ values of 6.59 and 5.79 , respectively. Débibakas et al. (2014) reported two markers associated with SCYLV at Chr02:4.6 Mbp and Chr08:62.4 Mbp in a sugarcane cultivar panel $(n=189)$. We had a marker (2P5229444) identified by four runs of the GWAS (with $-\log [P]$ ranging from 5.65 to 9.31 ) and a distance of $0.6 \mathrm{Mbp}$ from the QTL at chromosome 2. The closest marker (8P59311816) in this study to the QTL at chromosome 8 was $3.1 \mathrm{Mbp}$. In 2018, for SCYLV, Islam et al. (2018) identified two QTLs at Chr01:2.8 Mbp and Chr03:60.2 Mbp in a biparental population. We also identified strong associations for SCYLV resistance in nearby regions ( 0.9 and
A

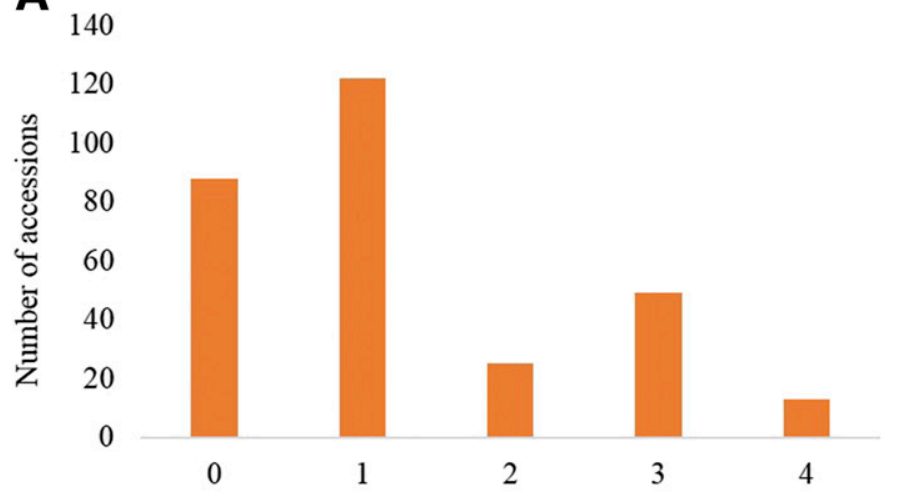

Average disease reactions of orange rust
B

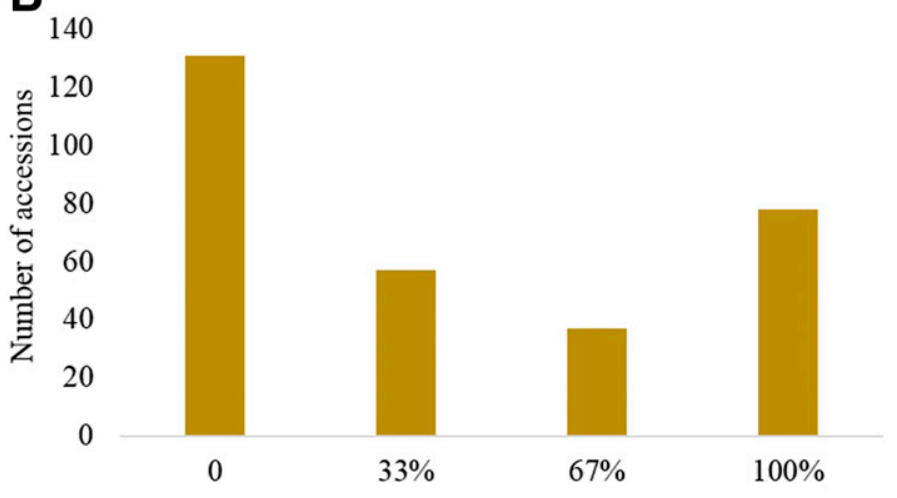

Average disease reactions of SCYLV
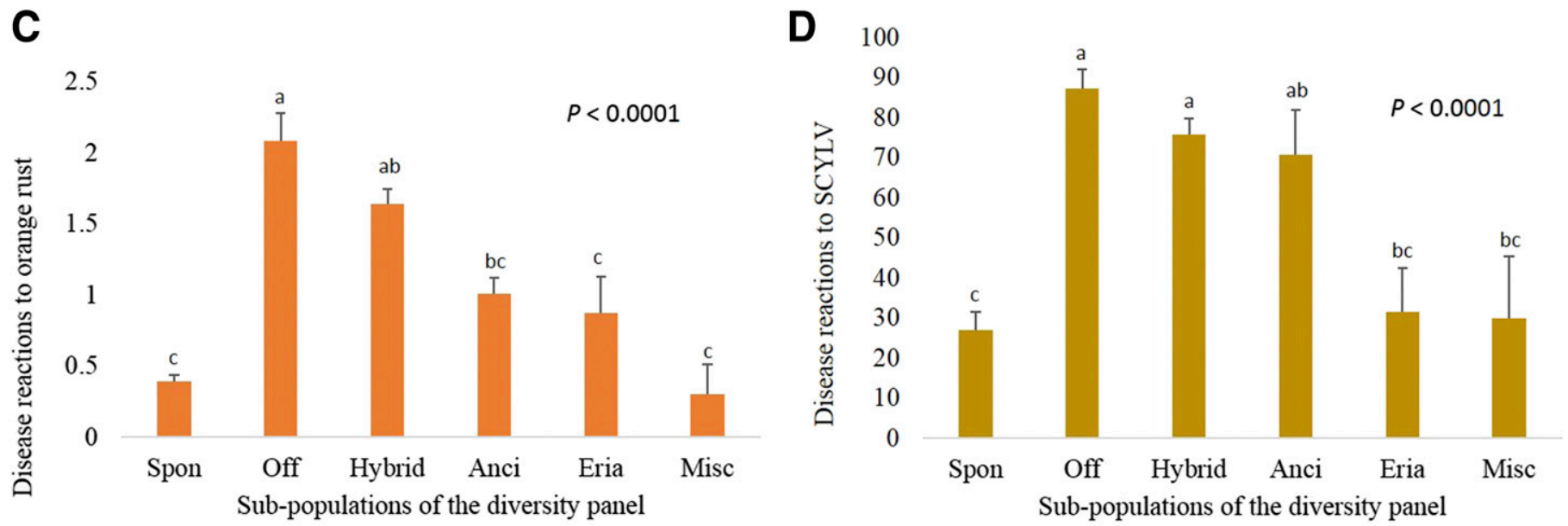

Fig. 2. Disease reactions in the sugarcane diversity panel. Distribution of average disease reactions to $\mathbf{A}$ and $\mathbf{C}$, sugarcane orange rust (SCOR) and $\mathbf{B}$ and $\mathbf{D}$, Sugarcane yellow leaf virus (SCYLV). The disease reactions are shown on a zero (the most resistant) to four (the most susceptible) scale for SCOR and as infection rate (0 to 100\%) for SCYLV. Error bars in C and D are standard errors of the mean for each subpopulation. Anci, ancient Saccharum hybrids, including Saccharum barberi and Saccharum sinence; Eria, the genus Erianthus; Hybrid, modern Saccharum hybrids; Misc, the genus Miscanthus; Off, Saccharum officinarum; Spon, Saccharum spontaneum. Tukey HSD test was applied to compare disease reactions to orange rust and SCYLV, separately. Different letters indicate significant different level at $P<0.05$. 
4.6 $\mathrm{Mbp}$ to the QTL at chromosomes 1 and 3, respectively). This comparison validated our GWAS results and suggested that we comprehensively identified a large number of novel associated markers.

Based on the phenotyping segregation patterns in this study, resistance to orange rust and SCYLV was quantitative in nature with contributions by multiple genes. Previous researchers (Costet et al. 2012; Débibakas et al. 2014; Islam et al. 2018; Yang et al. 2018a) and this study identified multiple QTLs or markers associated with resistance to the two diseases. This further endorses the theory that multiple genes are involved in sugarcane disease response. Moreover, additive effects were observed for orange rust and SCYLV resistance in previous reports (Débibakas et al. 2014; Yang et al. 2018a), whereas combining multiple resistant QTLs could significantly reduce disease incidence. We included dosage of markers and different gene actions in our GWAS analysis. Significant associations were identified under different models, supporting that various interactions existed among alleles, including additive, dominance, and dosage effects. For example, the marker (8P55553144) in the gene (Sobic.008G129700) showed that the alternative allele is dominant to the reference allele and that any dosage of this alternative allele could provide effective resistance to SCYLV (Fig. 5). The functions of associated candidate genes were diverse but highlighted in gene regulation, enzymatic activity, and receptor activity, suggesting that sugarcane responses to orange rust and SCYLV infections are quick and efficient by an action of integrating multiple levels of gene cooperation.
It is well known that there is concern about disease reactions to SCYLV, because no inoculation technique that is optimized is available for SCYLV at current stage. Thus, the disease index to SCYLV was probably impacted by aphid density and activity. In addition, the enzyme-linked immunosorbent assay used for SCYLV detection could show false negatives. In this study, all accessions of the diversity panel were regenerated from the WCSRG through vegetative propagation by cutting mature stalks, which were then maintained in pots with three replicates, and the evaluation of SCYLV was performed in ratoons the second year after the experimental trials were established. After an individual is infected with SCYLV, then the virus should remain in the stalks until clean treatment is conducted. We believe that all of the individuals had enough exposure to the virus (from accessions maintained in the WCSRG to the diversity panel established before SCYLV scoring). In addition, we did the disease evaluation on three biological replicates, which could reduce environmental variance. The high correlation coefficients (on average, 0.58 among the three replicates) and high broad sense heritability (0.80) supported that this study reduced variance caused by environments. Thus, we confidently assume that phenotypic data were collected properly for GWAS.

We used DAPC to assess the population structures of the sugarcane diversity using a previously established method (Yang et al. 2018b). Based on their discussion, DAPC was better than principal component analysis (PCA) in partitioning genetic variation into between-group and within-group components, and thus, it could achieve a better assignment of individuals into
A

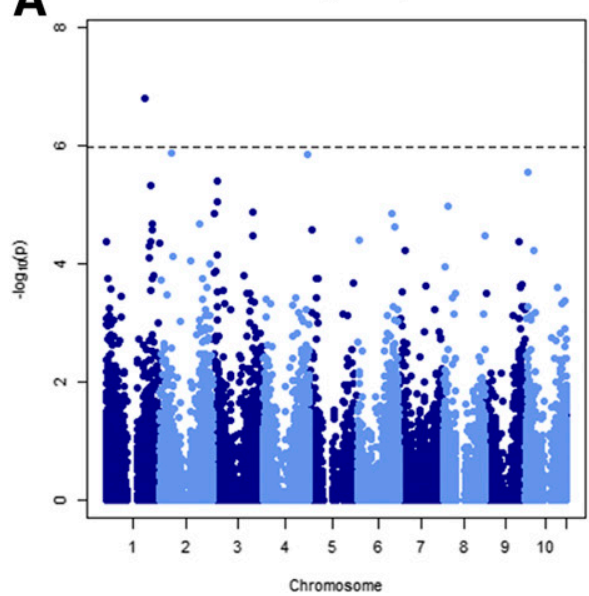

D

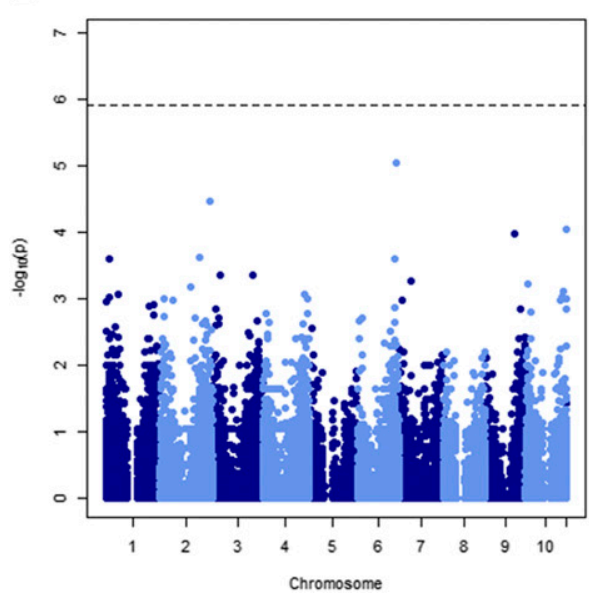

B

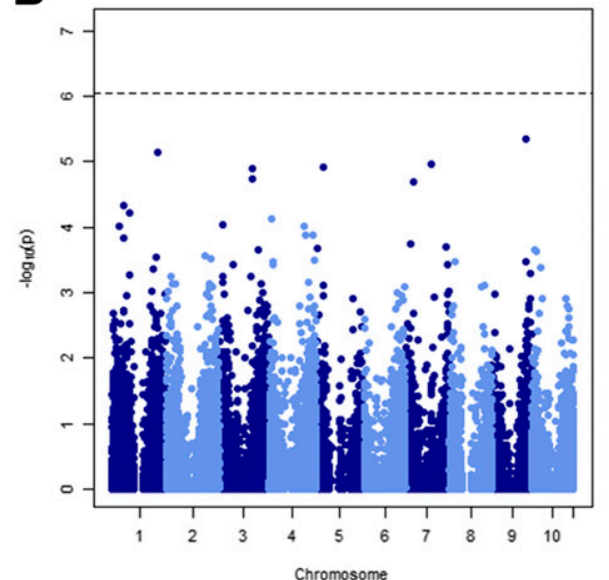

E

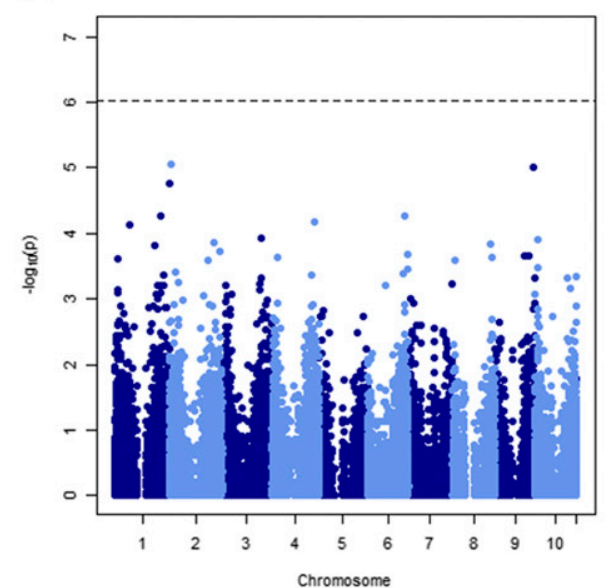

C

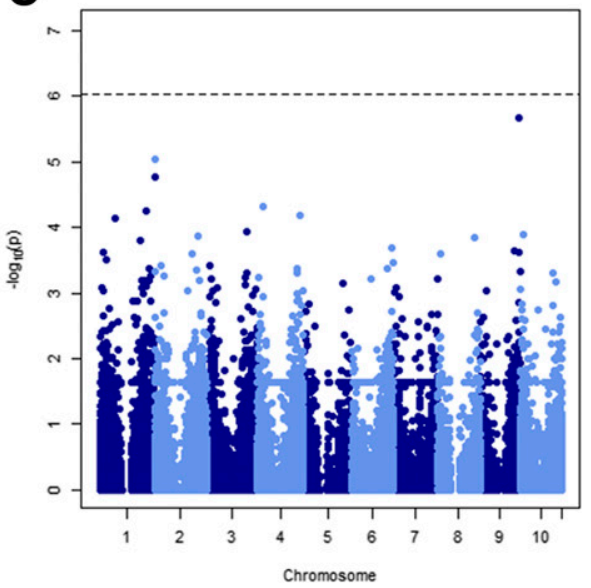

$\mathbf{F}$

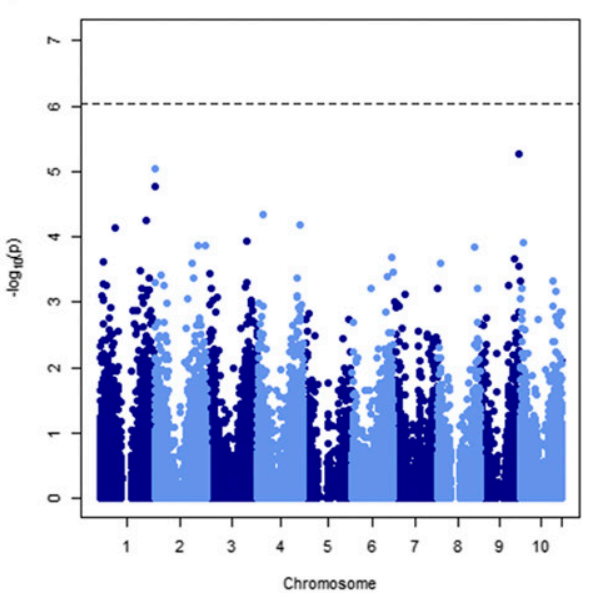

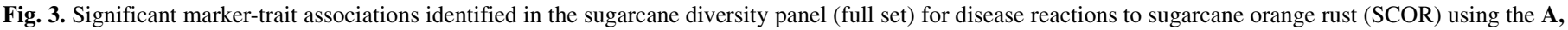
general model, B, additive model, C, 1-dom-alt model, D, 1-dom-ref model, E, diplo-general model, and F, diplo-additive model. 
predefined groups. In addition, DAPC was more suitable than Structure (Pritchard et al. 2000) and Admixture (Alexander et al. 2009) for sugarcane structure analysis because of the high polyploidy nature of sugarcane. Also, sugarcane outcrossing and clonal propagation do not fit the assumptions required for Structure and Admixture. In our study, the results from DAPC were consistent with grouping based on phylogenetic analysis. The Q-Q plots supported that the population structure estimated using DAPC worked well for all GWAS models, except for some suspicious inflations observed under the general model in the GWAS in $S$. spontaneum, which did not affect GWAS results. Therefore, we concluded that the population structures from DAPC were reliable and suitable for subsequent GWAS in this study.

The sugarcane diversity panel for disease research. There were several studies conducted in sugarcane to identify genes/markers controlling disease resistance to orange rust and SCYLV (Costet et al. 2012; Débibakas et al. 2014; Islam et al. 2018; Yang et al. 2018a). However, either these studies were performed in a biparental population, or elite germplasm was assembled from primarily parental clones and sugarcane hybrids. Their strategies could characterize alleles segregating in modern sugarcane hybrids that possibly could be quickly utilized for sugarcane improvement. However, it is well known that modern sugarcane cultivars have a narrow genetic basis because of only a few foundation accessions involved in their initial hybridizations (Acevedo et al. 2017; Deren 1995; Lima et al. 2002). Therefore, various novel but important genetic components are unlikely to be identified in those narrow populations. It is necessary to collect a large and diverse sugarcane panel for the GWAS to identify novel marker trait associations, especially for finding sources of disease resistance, which is mainly inherited by sugarcane from the wild species, $S$. spontaneum.

This study took advantage of the WCSRG, which was originally collected from 45 different countries in nine geographical regions

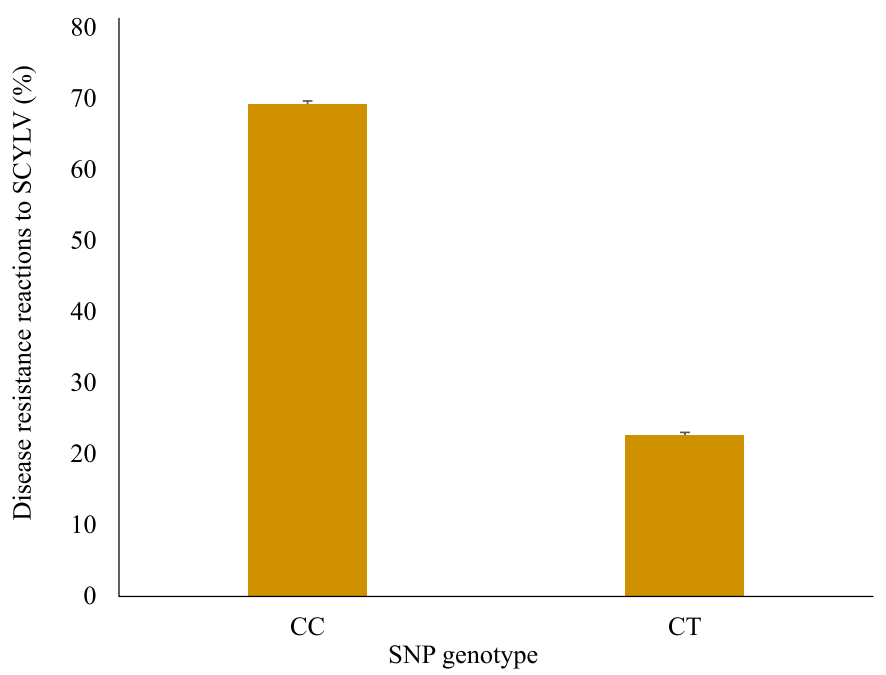

Fig. 5. Disease reactions to Sugarcane yellow leaf virus (SCYLV) according to the associated single-nucleotide polymorphism (SNP; 8P55553144), which is located in the gene (Sobic.008G129700). Error bar is the standard error of the mean for each marker genotype. $\mathrm{C}$ is the reference allele, whereas $\mathrm{T}$ is alternative allele.
A

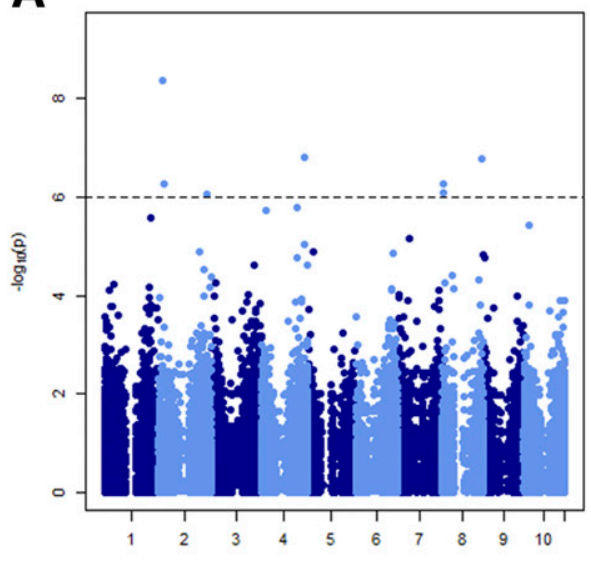

Chromosome

D

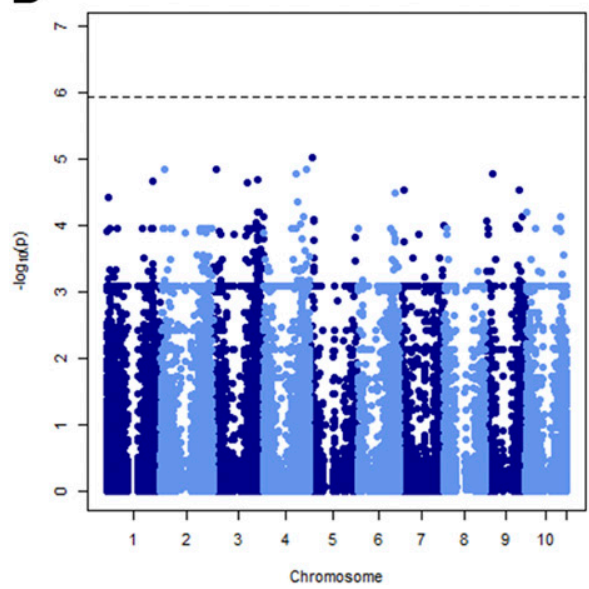

B

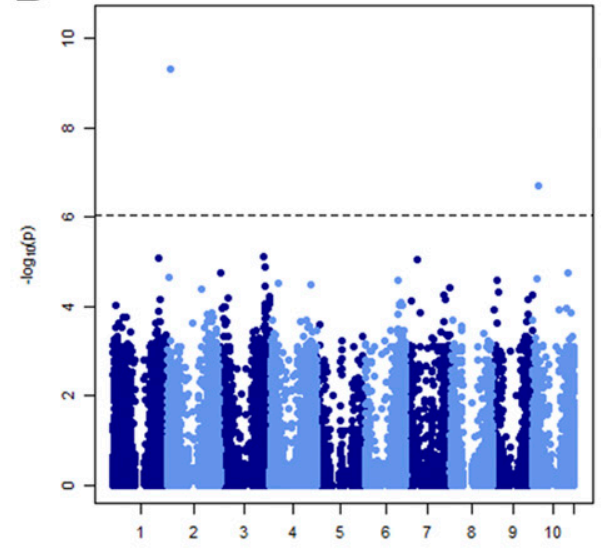

Chromosome

E

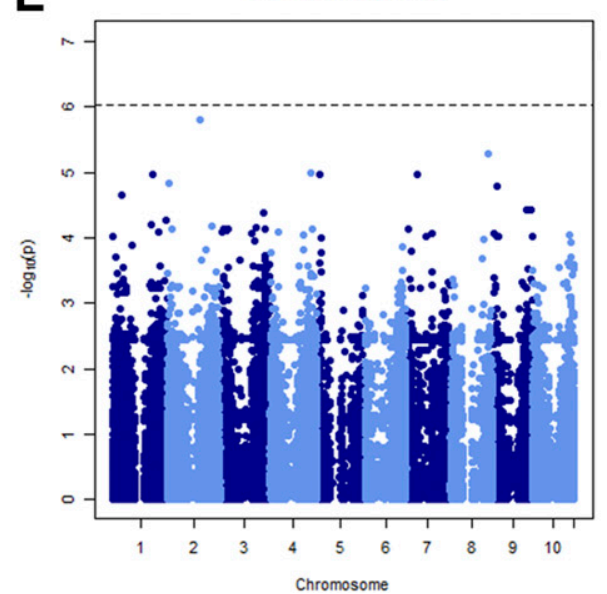

C

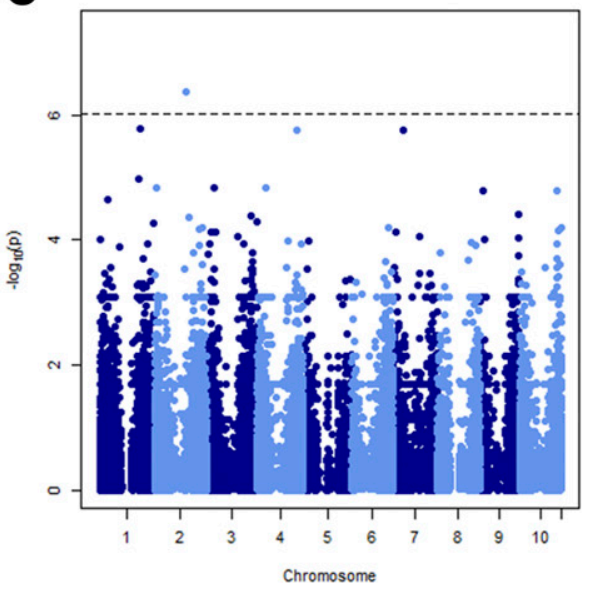

$\mathbf{F}$

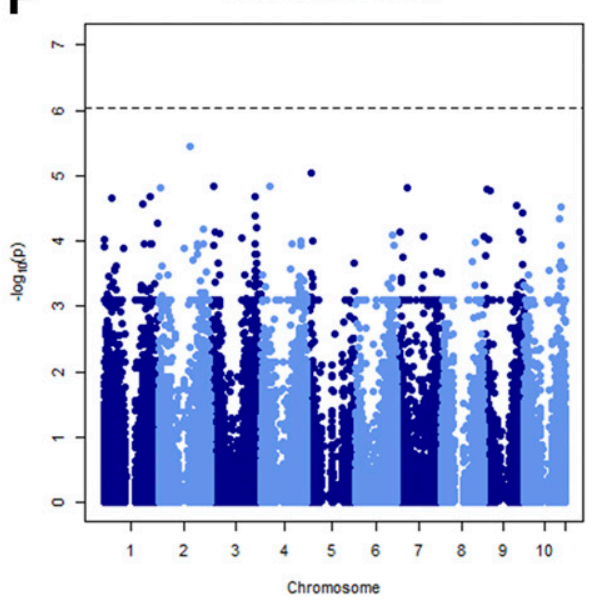

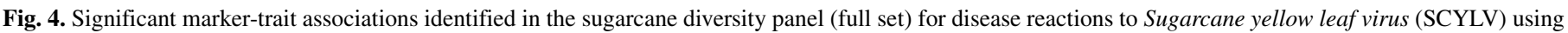
the A, general model, B, additive model, C, 1-dom-alt model, D, 1-dom-ref model, E, diplo-general model, and F, diplo-additive model. 
(Nayak et al. 2014; Todd et al. 2014); from it, a representative panel was assembled, including 279 Saccharum accessions and 29 nonSaccharum accessions. Our results showed a significant amount of genetic and phenotypic diversity in the diversity panel, which can be used for identification of genetic resources controlling disease resistance in sugarcane. First, the analyses of sequence variants in the diversity panel supported that interspecies hybridization led to a relatively high genetic diversity in Saccharum hybrids compared with S. officinarum (Table 1). However, a large portion of genetic resources from $S$. spontaneum and $S$. officinarum was likely not included into modern Saccharum hybrids. For example, focusing on conserved regions in the 30 accessions analyzed, 23.5 and $11.3 \%$ of alleles from $S$. spontaneum and $S$. officinurum, respectively, were not present in modern Saccharum hybrids. These percentages would increase substantially by taking into consideration regions with structure variations. Therefore, large amounts of genetic resources with allelic segregation were probably only present in $S$. spontaneum, especially disease resistance resources. Second, based on the evaluation of disease resistance, we found that disease reactions segregated well in the diversity panel, and $S$. spontaneum and non-Saccharum species were more resistant to orange rust and SCYLV diseases compared with $S$. officinarum and modern sugarcane hybrids. As expected, more markers were identified from the GWAS in S. spontaneum, whereas few associations were identified from sugarcane hybrids or S. officinurum.

This study strongly suggested that diverse germplasm (sources other than Saccharum hybrids) should be substantially utilized for sugarcane cultivar improvement. Resistance traits can be quickly introduced into sugarcane cultivars by controlled crosses with the help of tightly linked molecular markers, because the Saccharum species are readily intercrossed to produce fertile hybrids, and nonSaccharum species were also often reported to be used in sugarcane breeding programs (Aitken et al. 2007; Cai et al. 2005; Chen et al. 1983; Clifton-Brown et al. 2008; Wang et al. 2009). In fact, this process to breed resistant cultivars will be significantly improved by using molecular markers in selection, which were identified in this study and previous studies.

\section{ACKNOWLEDGMENTS}

We thank B. Glaz for carefully reviewing the revised manuscript.

\section{LITERATURE CITED}

Acevedo, A., Tejedor, M. T., Erazzú, L. E., Cabada, S., and Sopena, R. 2017. Pedigree comparison highlights genetic similarities and potential industrial values of sugarcane cultivars. Euphytica 213:121.

Aitken, K., Li, J., Wang, L., Qing, C., Fan, Y. H., and Jackson, P. 2007. Characterization of intergeneric hybrids of Erianthus rockii and Saccharum using molecular markers. Genet. Resour. Crop Evol. 54:1395-1405.

Alexander, D. H., Novembre, J., and Lange, K. 2009. Fast model-based estimation of ancestry in unrelated individuals. Genome Res. 9:1655-1664.

Bolger, A. M., Lohse, M., and Usadel, B. 2014. Trimmomatic: A flexible trimmer for Illumina sequence data. Bioinformatics 30:2114-2120.

Cai, Q., Aitken, K., Deng, H. H., Chen, X. W., Fu, C., Jackson, P. A., and McIntyre, C. L. 2005. Verification of the introgression of Erianthus arundinaceus germplasm into sugarcane using molecular markers. Plant Breed. 124:322-328.

Chen, W. H., Huang, Y. J., Shen, I. S., and Shih, S. C. 1983. Utilization of Miscanthus germplasm in sugarcane breeding in Taiwan. Proc. Int. Soc. Sugarcane Technol. 18:641-648.

Clifton-Brown, J., Chiang, Y., and Hodkinson, T. R. 2008. Miscanthus: Genetic resources and breeding potential to enhance bioenergy production. Pages 273-294 in: Genetic Improvement of Bioenergy Crops. W. Vermerris, ed. Springer Science \& Business Media LLC, New York.

Costet, L., Raboin, L., Payet, M., D'Hont, A., and Nibouche, S. 2012. A major quantitative trait allele for resistance to the Sugarcane yellow leaf virus (Luteoviridae). Plant Breed. 131:637-640.

Dahlquist, E., ed. 2013. Biomass as Energy Source: Resources, Systems and Applications. CRC Press, Taylor \& Francis, London, United Kingdom.

Daugrois, J. H., Edon-Jock, C., Bonoto, S., Vaillant, J., and Rott, P. 2011. Spread of Sugarcane yellow leaf virus in initially disease-free sugarcane is linked to rainfall and host resistance in the humid tropical environment of Guadeloupe. Eur. J. Plant Pathol. 129:71-80.

Débibakas, S., Rocher, S., Garsmeur, O., Toubi, L., Roques, D., D’Hont, A., Hoarau, J., and Daugrois, J. 2014. Prospecting sugarcane resistance to sugarcane yellow leaf virus by genome-wide association. Theor. Appl. Genet. 127:1719-1732.

Deren, C. W. 1995. Genetic base of US mainland sugarcane. Crop Sci. 35: 1195-1199.

Diévart, A., Gilbert, N., Droc, G., Attard, A., Gourgues, M., Guiderdoni, E., and Périn, C. 2011. Leucine-rich repeat receptor kinases are sporadically distributed in eukaryotic genomes. BMC Evol. Biol. 11:367.

FAOSTAT. 2016. FAOSTAT, FAO Statistical Databases. http://www.fao.org/ faostat/en/\#home

Gonçalves, M. C., Vega, J., Oliveira, J. G., and Gomes, M. 2005. Sugarcane yellow leaf virus infection leads to alterations in photosynthetic efficiency and carbohydrate accumulation in sugarcane leaves. Fitopatol. Bras. 30:10-16.

Harrell, F. E., Jr. (2018) Hmisc: Harrell miscellaneous. https://cran.r-project. org/web/packages/Hmisc/index.html

Islam, M. S., Yang, X., Sood, S., Comstock, J. C., and Wang, J. 2018. Molecular characterization of genetic basis of Sugarcane Yellow Leaf Virus (SCYLV) resistance in Saccharum spp. hybrid. Plant Breed. 137:598-604.

Jombart, T. 2008. adegenet: A R package for the multivariate analysis of genetic markers. Bioinformatics 24:1403-1405.

Jombart, T., Devillard, S., and Balloux, F. 2010. Discriminant analysis of principal components: A new method for the analysis of genetically structured populations. BMC Genet. 11:94.

Jones, P., Binns, D., Chang, H. Y., Fraser, M., Li, W., McAnulla, C., McWilliam, H., Maslen, J., Mitchell, A., Nuka, G., Pesseat, S., Quinn, A. F., Sangrador-Vegas, A., Scheremetjew, M., Yong, S. Y., Lopez, R., and Hunter, S. 2014. InterProScan 5: Genome-scale protein function classification. Bioinformatics 30:1236-1240.

Li, H. 2013. Aligning sequence reads, clone sequences and assembly contigs with BWA-MEM. arXiv:1303.3997.

Lima, M. L. A., Garcia, A. A. F., Oliveira, K. M., Matsuoka, S., Arizono, H., de Souza C. L., Jr., and de Souza, A. P. 2002. Analysis of genetic similarity detected by AFLP and coefficient of parentage among genotypes of sugar cane (Saccharum spp.). Theor. Appl. Genet. 104:30-38.

Lyne R., Sullivan J., Butano D., Contrino S., Heimbach J., Hu F., Kalderimis A., Lyne M., N. Smith, R., Štěpán, R., Balakrishnan, R., Binkley, G., Harris, T., Karra, K., A. T. Moxon, S., Motenko, H., Neuhauser, S., Ruzicka, L., Cherry, M., Richardson, J., Stein, L., Westerfield, M., Worthey, E., and Micklem, G. 2015. Cross-organism analysis using InterMine. Genesis 53: 547-560.

Magarey, R. C., Royal, A., Williams, D. J., and Bull, J. I. 2011. A brief history of disease epidemics in Queensland and of some economic outcomes. Proc. Aust. Soc. Sugar Cane Technol. 33:1-12.

McKenna, A., Hanna, M., Banks, E., Sivachenko, A., Cibulskis, K., Kernytsky, A., Garimella, K., Altshuler, D., Gabriel, S., and Daly, M. 2010. The Genome Analysis Toolkit: A MapReduce framework for analyzing nextgeneration DNA sequencing data. Genome Res. 20:1297-1303.

Moonan, F., Molina, J., and Mirkov, T. E. 2000. Sugarcane yellow leaf virus: An emerging virus that has evolved by recombination between luteoviral and poleroviral ancestors. Virology 269:156-171.

Nayak, S. N., Song, J., Villa, A., Pathak, B., Ayala-Silva, T., Yang, X., Todd, J., Glynn, N. C., Kuhn, D. N., and Glaz, B. 2014. Promoting utilization of Saccharum spp. genetic resources through genetic diversity analysis and core collection construction. PLoS One 9:e110856.

Paterson, A. H., Bowers, J. E., Bruggmann, R., Dubchak, I., Grimwood, J., Gundlach, H., Haberer, G., Hellsten, U., Mitros, T., Poliakov, A., Schmutz, J., Spannagl, M., Tang, H., Wang, X., Wicker, T., Bharti, A. K., Chapman, J., Feltus, F. A., Gowik, U., Grigoriev, I. V., Lyons, E., Maher, C. A., Martis, M., Narechania, A., Otillar, R. P., Penning, B. W., Salamov, A. A., Wang, Y., Zhang, L., Carpita, N. C., Freeling, M., Gingle, A. R., Hash, C. T., Keller, B., Klein, P., Kresovich, S., McCann, M. C., Ming, R., Peterson, D. G., Mehboob-ur-Rahman, Ware, D., Westhoff, P., Mayer, K. F. X., Messing, J., and Rokhsar, D. S. 2009. The Sorghum bicolor genome and the diversification of grasses. Nature 457:551-556.

Pinheiro, J., Bates, D., DebRoy, S., and Sarkar, D. 2014. Linear and nonlinear mixed effects models. $\mathrm{R}$ package version 3:1-117.

Pritchard, J. K., Stephens, M., and Donnelly, P. 2000. Inference of population structure using multilocus genotype data. Genetics 2:945-959.

Rassaby, L., Girard, J., Lemaire, O., Costet, L., Irey, M. S., Kodja, H., Lockhart, B. E., and Rott, P. 2004. Spread of Sugarcane yellow leaf virus in sugarcane plants and fields on the island of Réunion. Plant Pathol. 53: $117-125$

Rassaby, L., Girard, J., Letourmy, P., Chaume, J., Irey, M. S., Lockhart, B. E., Kodja, H., and Rott, P. 2003. Impact of Sugarcane yellow leaf virus on sugarcane yield and juice quality in Réunion Island. Eur. J. Plant Pathol. 109:459-466. 
Rosyara, U. R., De Jong, W. S., Douches, D. S., and Endelman, J. B. 2016. Software for genome-wide association studies in autopolyploids and its application to potato. Plant Genome 9:1-10.

Schenck S. 1997. Use of a tissue blot immunoassay to determine the distribution of Sugarcane yellow leaf virus in Hawaii. Sugar Cane 4:5-8.

Song, J., Yang, X., Resende, M. F., Jr., Neves, L. G., Todd, J., Zhang, J., Comstock, J. C., and Wang, J. 2016. Natural allelic variations in highly polyploidy Saccharum complex. Front. Plant Sci. 7:804.

Sood, S. G., Comstock, J. C., and Glynn, N. C. 2009. Leaf whorl inoculation method for screening sugarcane rust resistance. Plant Dis. 93:1335-1340.

Todd, J., Wang, J., Glaz, B., Sood, S., Ayala-Silva, T., Nayak, S. N., Glynn, N. C., Gutierrez, O. A., Kuhn, D. N., and Tahir, M. 2014. Phenotypic characterization of the Miami World Collection of sugarcane (Saccharum spp.) and related grasses for selecting a representative core. Genet. Resour. Crop Evol. 61:1581-1596.

Wang, J., Roe, B., Macmil, S., Yu, Q., Murray, J. E., Tang, H., Chen, C., Najar, F., Wiley, G., and Bowers, J. 2010. Microcollinearity between autopolyploid sugarcane and diploid sorghum genomes. BMC Genomics 11:261.

Wang, X.-H., Yang, Q.-H., Li, F.-S., He, L.-L., and He, S.-C. 2009. Molecular identification of Saccharum spp. x Erianthus fulvus hybrids using sequencecharacterized amplified region markers. Crop Sci. 49:864-870.

Yang, X., Islam, M. S., Sood, S., Maya, S., Hanson, E. A., Comstock, J., and Wang, J. 2018a. Identifying quantitative trait loci (QTLs) and developing diagnostic markers linked to orange rust resistance in sugarcane (Saccharum spp.). Front. Plant Sci. 9:350.

Yang, X., Luo, Z., Todd, J., Sood, S., and Wang, J. 2018b. Genome-wide association study of multiple yield components in a diversity panel of polyploid sugarcane (Saccharum spp.). bioRxiv:387001.

Yang, X., Song, J., Todd, J., Peng, Z., Paudel, D., Luo, Z., Ma, X., You, Q., Hanson, E., Zhao, Z., Zhao, Y., Zhang, J., Ming, R., and Wang, J. 2019. Target enrichment sequencing of 307 germplasm accessions identified ancestry of ancient and modern hybrids and signatures of adaptation and selection in sugarcane (Saccharum spp.), a "sweet" crop with "bitter" genomes. Plant Biotechnol. J. 17:488-498.

Yang, X., Song, J., You, Q., Paudel, D. R., Zhang, J., and Wang, J. 2017. Mining sequence variations in representative polyploid sugarcane germplasm accessions. BMC Genomics 18:594.

Yang X., and Wang J. 2016. Genome-wide analysis of NBS-LRR genes in sorghum genome revealed several events contributing to NBS-LRR gene evolution in grass species. Evol. Bioinform. Online 12:9-21.

Yi, X., Du, Z., and Su, Z. 2013. PlantGSEA: A gene set enrichment analysis toolkit for plant community. Nucleic Acids Res. 41:W98-W103.

Zhao, D., Glynn, N. C., Glaz, B., Comstock, J. C., and Sood, S. 2011. Orange rust effects on leaf photosynthesis and related characters of sugarcane. Plant Dis. 95:640-647. 